\title{
Natural orifice transluminal endoscopic mediastinal surgery: NOTEMS, a promising field for endotherapy
}

The mediastinum used to be a forbidden area for endotherapy because of its complicated and variable anatomical structure [1]. Traditional surgery is regarded as curative but brings with it great trauma, a long recovery, high costs, and a poor quality of life [2]. To overcome these disadvantages, in our previous work we reported a novel approach to paraesophageal structures using the narrow submucosal tunnel or "third space" as an operating entry into the mediastinal cavity [3]. However, we still encountered several problems. When tumors are located higher than $22 \mathrm{~cm}$ from the incisors, it is impossible to start the tunnel entry $5 \mathrm{~cm}$ above the tumor site. In addition, when the tumor is larger than $5 \mathrm{~cm}$ in diameter, it cannot be taken out through the tunnel en bloc without injuring the mucosa, but has to be removed piecemeal instead. We are eager to update endoscopic procedures to improve the approach to such tumors.

Herein, we report on a novel technique, called natural orifice transluminal endoscopic mediastinal surgery (NOTEMS), which involves full-thickness resection of the esophageal wall to dissect the tumor in the mediastinal cavity, followed by closure of the wound by metallic clips along with biological glue, to treat large mediastinal tumors around the upper esophagus (> Fig.1). Using NOTEMS, we successfully treated a 30-year-old patient admitted because of an esophageal submucosal tumor (SMT) found on routine check-up by regular esophagogastroduodenoscopy (EGD) ( $\vee$ Fig. 2). By means of computed tomography of the chest and endoscopic ultrasonography the SMT was diagnosed as a giant posterosuperior

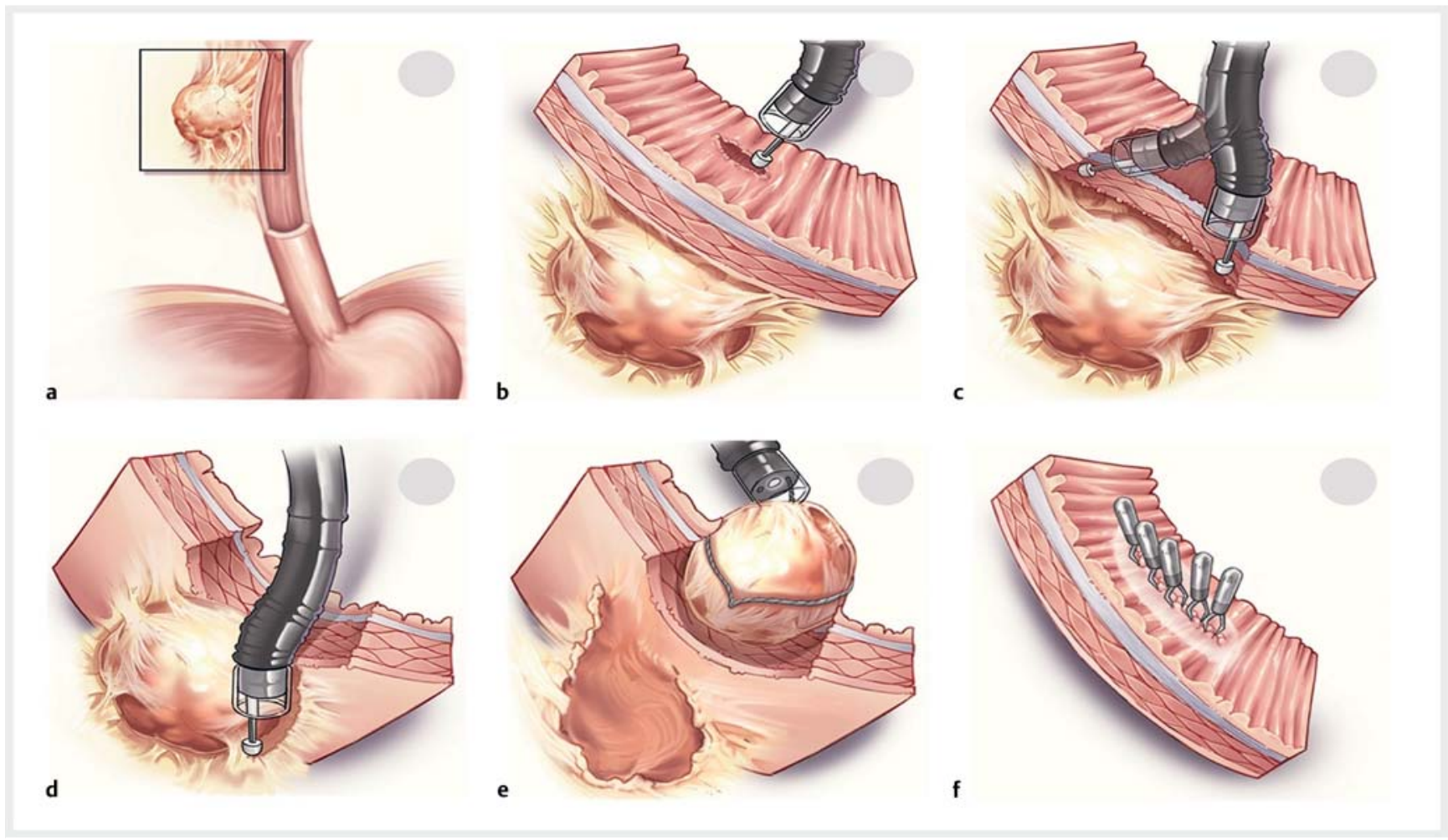

Fig. 1 The NOTEMS technique. The patient is under general anesthesia with endotracheal intubation and prophylactic intravenous antibiotics introduced preoperatively. The procedure involves five steps as follows: a, b Step 1: Mucosal incision. Locate the tumor site and perform a submucosal injection followed by a 2-cm longitudinal mucosal incision made right above the tumor site. c Step 2: Full-thickness resection of the esophageal wall. Incise the esophageal wall layer by layer from the mucosa, submucosa to muscularis, and expose the tumor in the mediastinal cavity, then extend the incision along the vertical diameter of the tumor to ensure the tumor can be removed smoothly. d Step 3: Tumor dissection. Dissect the surrounding tissues as close as possible to the tumor capsule but still with the aim of achieving en bloc resection. e Step 4: Tumor extraction. Using a snare, extract the tumor from the mediastinum directly into the esophageal cavity and out of the patient, then inspect the wound cavity carefully for any evidence of bleeding sites. $\mathbf{f}$ Step 5: Closure of the mucosal incision. Close the mucosal incision above the cavity left behind by placing several metallic clips and spraying biological glue. 

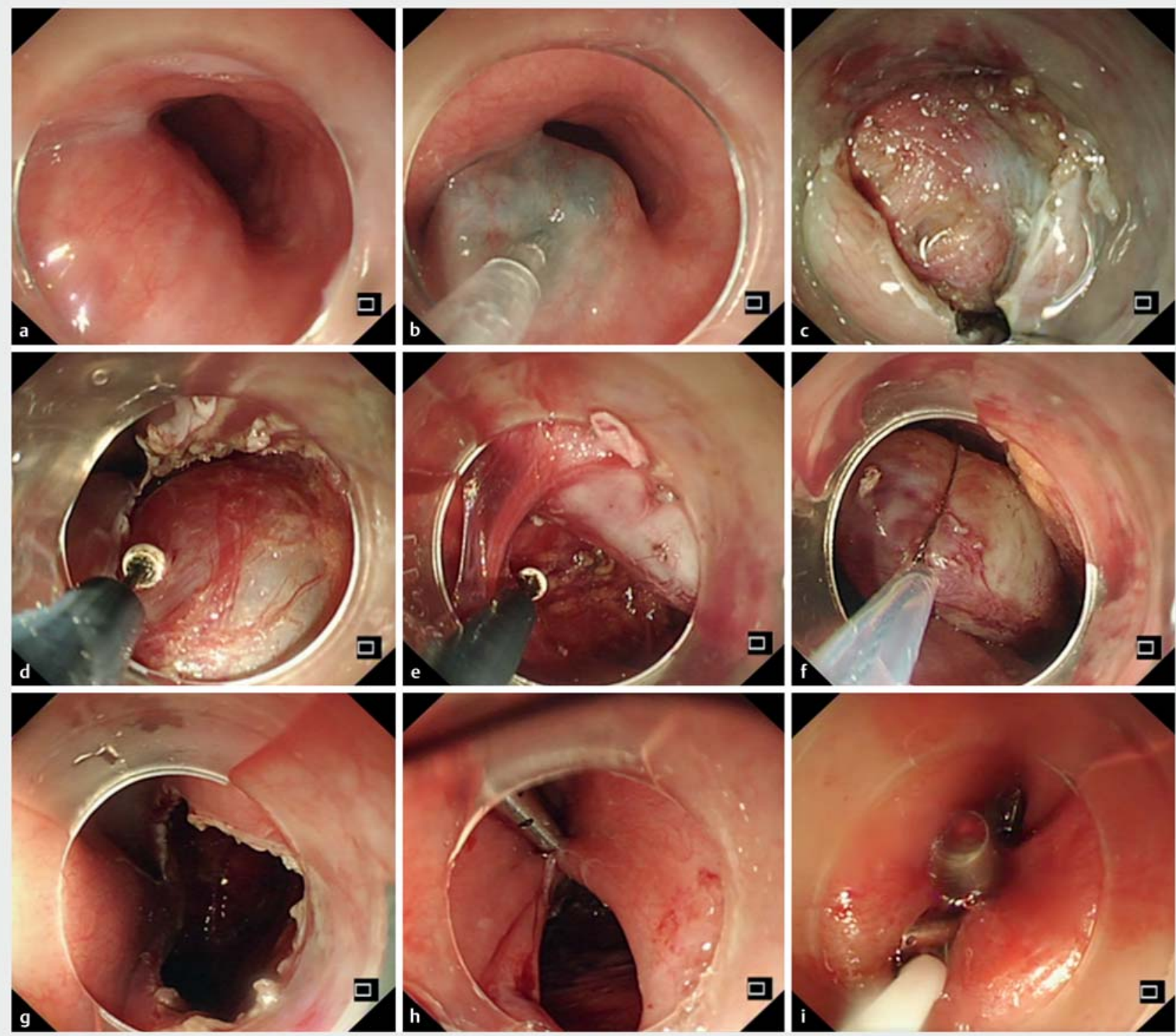

- Fig. 2 NOTEMS for the treatment of a benign mediastinal tumor in a 30-year-old young man. The procedure included five steps as follows: a visualization of a giant tumor $21-26 \mathrm{~cm}$ from the incisors; $\mathbf{b}$ mucosal incision; $\mathbf{c}$ full-thickness resection of the esophageal wall; $\mathbf{d}$ extension of the incision along the vertical diameter of the tumor to ensure the tumor could be removed smoothly; e dissection of the surrounding tissue; $\mathbf{f}$ tumor dissection and extraction using a snare; $\mathbf{g}$ careful inspection of the wound cavity for any evidence of bleeding sites; $\mathbf{h}$, $\mathbf{i}$ closure of the mucosal incision by placing of several metallic clips and spraying biological glue.

mediastinal tumor, and the patient very much wanted it removed ( $\triangleright$ Video 1 ). Pathologic analysis showed a mixture of neurofibroma and schwannoma. The patient presented with rebound hyperthermia on postoperative day (POD) 1 , which was relieved by intravenous antibiotics given for 4 days. On POD6 endoscopic examination showed metallic clips in place and wound healing with glue on top ( Fig.3), and on POD7 the patient was discharged. Routine follow-up EGD confirmed good healing.
Endoscopy_UCTN_Code_TTT_1AO_2AN

\section{Funding}

National Natural Science Foundation of China

http://dx.doi.org/10.13039/

501100001809

81902394

National Natural Science Foundation of China

http://dx.doi.org/10.13039/

501100001809

82000623
National Natural Science Foundation of China

http://dx.doi.org/10.13039/

501100001809

82003074 

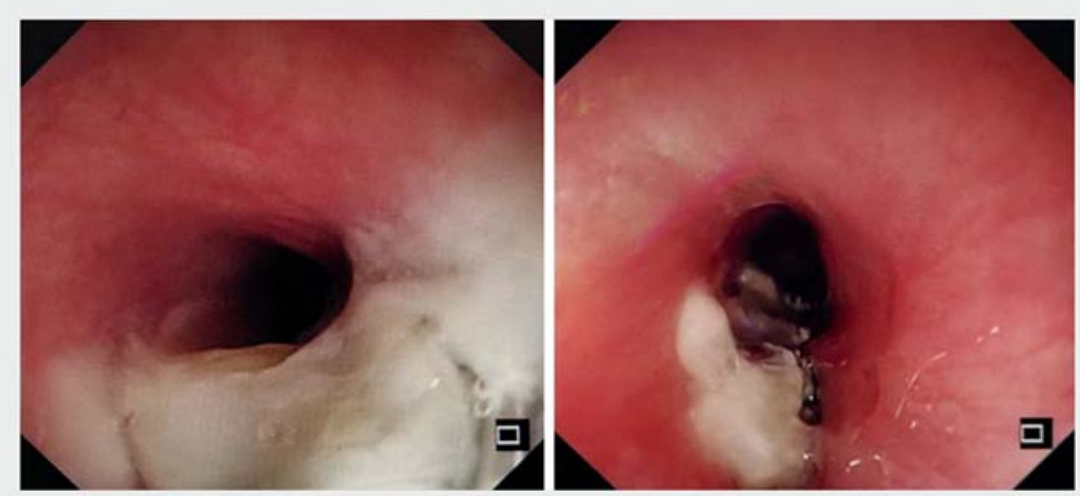

- Fig. 3 On postoperative day 6 , endoscopic examination showed metallic clips in place and wound healing with glue on top.

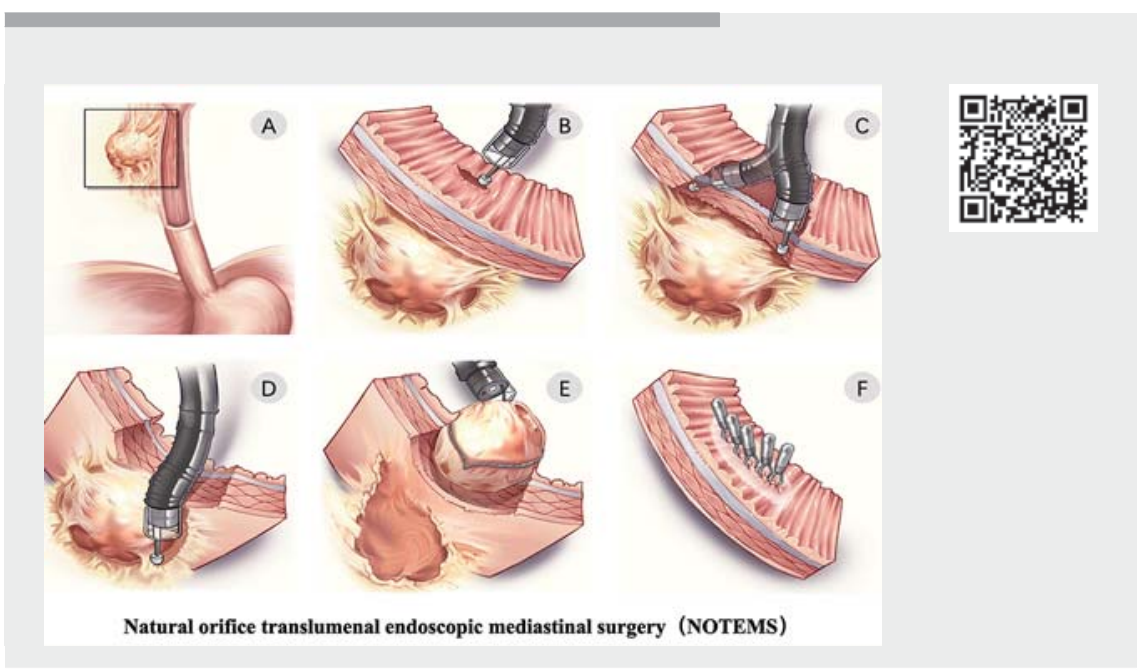

$\checkmark$ Video 1 Natural orifice transluminal endoscopic mediastinal surgery (NOTEMS) for the treatment of a benign mediastinal tumor in a 30 -year-old young man.

\section{Competing interests}

The authors declare that they have no conflict of interest.

The authors

Ping-ting Gao*, Sheng-li Lin*, Pei-yao Fu, Xinyang Liu, Quan-lin Li, Wei-feng Chen, Pinghong Zhou

Endoscopy Center and Endoscopy Research Institute, Zhongshan Hospital, Fudan University, Shanghai, China
[1] den Bakker MA, Marx A, Mukai K et al. Mesenchymal tumours of the mediastinum - part I. Virchows Arch 2015; 467: 487-500

[2] Petty JK, Bensard DD, Partrick DA et al. Resection of neurogenic tumors in children: is thoracoscopy superior to thoracotomy? J Am Coll Surg 2006; 203: 699-703

[3] Gao P, Li Q, Hu J et al. Transoesophageal endoscopic removal of a benign mediastinal tumour: a new field for endotherapy? Gut 2020; 69: 1727-1729

Bibliography

Endoscopy 2022; 54: E357-E359

DOI 10.1055/a-1540-5732

ISSN 0013-726X

published online 9.8.2021

(c) 2021. Thieme. All rights reserved.

Georg Thieme Verlag KG, Rüdigerstraße 14,

70469 Stuttgart, Germany

\section{ENDOSCOPY E-VIDEOS \\ https://eref.thieme.de/e-videos}

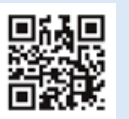

Endoscopy E-Videos is an open access online section, reporting on interesting cases and new techniques in gastroenterological endoscopy. All papers include a high quality video and all contributions are freely accessible online. Processing charges apply (currently EUR 375), discounts and wavers acc. to HINARI are available.

This section has its own submission website at https://mc.manuscriptcentral.com/e-videos

\section{Ping-hong Zhou, MD, PhD}

Endoscopy Center and Endoscopy Research Institute, Zhongshan Hospital, Fudan University, 180 FengLin Road, Shanghai 200032, P. R. China zhou.pinghong@zs-hospital.sh.cn

\footnotetext{
* Ping-ting Gao and Sheng-li Lin contributed equally to this work.
} 